\title{
Global reconnection topology as inferred from plasma observations inside Kelvin-Helmholtz vortices
}

\author{
M. B. Bavassano Cattaneo ${ }^{1}$, M. F. Marcucci ${ }^{1}$, Y. V. Bogdanova ${ }^{2}$, H. Rème ${ }^{3}$, I. Dandouras ${ }^{3}$, L. M. Kistler ${ }^{4}$, and \\ E. Lucek $^{5}$ \\ ${ }^{1}$ Istituto di Fisica dello Spazio Interplanetario, Istituto Nazionale di Astrofisica, Roma, Italy \\ ${ }^{2}$ Mullard Space Science Laboratory, University College London, Dorking, UK \\ ${ }^{3}$ CESR, Toulouse, France \\ ${ }^{4}$ University of New Hampshire, Durham, USA \\ ${ }^{5}$ Blackett Laboratory, Imperial College, London, UK
}

Received: 9 July 2009 - Revised: 5 February 2010 - Accepted: 19 March 2010 - Published: 1 April 2010

\begin{abstract}
During a long lasting period of northward interplanetary magnetic field and high solar wind speed (above $700 \mathrm{~km} / \mathrm{s}$ ), the Cluster spacecraft go across a number of very large rolled-up Kelvin-Helmholtz (KH) vortices at the dusk magnetopause, close to the terminator. The peculiarity of the present event is a particular sequence of ions and electrons distribution functions observed repeatedly inside each vortex. In particular, whenever Cluster crosses the current layer inside the vortices, multiple field-aligned ion populations appear, suggesting the occurrence of reconnection. In addition, the ion data display a clear velocity filter effect both at the leading and at the trailing edge of each vortex. This effect is not present in the simultaneous electron data. Unlike other $\mathrm{KH}$ studies reported in the literature in which reconnection occurs within the vortices, in the present event the observations are not compatible with local reconnection, but are accounted for by lobe reconnection occurring along an extended X-line at the terminator in the Southern Hemisphere. The reconnected field lines "sink" across the magnetopause and then convect tailward-duskward where they become embedded in the vortices. Another observational evidence is the detected presence of solar wind plasma on the magnetospheric side of the vortices, which confirms unambiguously the occurrence of mass transport across the magnetopause already reported in the literature. The proposed reconnection scenario accounts for all the observational aspects, regarding both the transport process and the kinetic signatures.
\end{abstract}

\section{Correspondence to:}

M. B. Bavassano Cattaneo

(maria.bice.cattaneo@ifsi-roma.inaf.it)
Keywords. Magnetospheric physics (Magnetopause, cusp, and boundary layers; Solar wind-magnetosphere interactions) - Space plasma physics (Magnetic reconnection)

\section{Introduction}

The Kelvin-Helmholtz instability (KHI) on the flanks of the magnetopause (MP) is considered one of the mechanisms for populating the low latitude boundary layer (LLBL) during periods of northward interplanetary magnetic field (IMF), when reconnection at the equatorial magnetopause is less effective. The condition for onset of KHI is a large velocity shear across the MP. Many observations of KH vortices on the flanks of the MP have been reported: since the early observations by Hones et al. (1981), many observations followed: e.g. Chen and Kivelson (1993) detected non sinusoidal surface waves, Fairfield et al. (2000) analysed a Geotail event and, with the help of adapted MHD simulations by Otto and Fairfield (2000), characterized the various regions in the vortices. Hasegawa et al. (2004) in a KH event, thanks to the multipoint capability of Cluster, showed clear evidence of plasma transport across the MP which they attributed to rolled-up KH vortices. Foullon et al. (2008) characterized the evolution of $\mathrm{KH}$ wave activity in a prolonged interval of northward IMF. Other aspects of KH vortices properties have been also extensively investigated by numerical simulations (e.g. Miura and Pritchett, 1982; Miura, 1995, 1999; Otto and Fairfield, 2000; Nakamura and Fujimoto, 2005; Nakamura et al., 2006). Simulations by Hashimoto and Fujimoto (2006) and by Takagi et al. (2006) have shown that

Published by Copernicus Publications on behalf of the European Geosciences Union. 
in rolled-up vortices non-MHD effects (such as formation of smaller scale structures or reconnection inside the vortices) may become important thus allowing plasma transport across the MP to occur.

From the kinetic point of view, in passing through $\mathrm{KH}$ vortices, typically the ions distibution function switches from magnetosheath-like to magnetospheric-like features. There are, however, cases in which the kinetic signatures are more complex and evidence the occurrence of magnetic reconnection inside the vortices, which had been predicted by numerical studies such as e.g. Otto and Fairfield (2000); Nakamura and Fujimoto (2005); and Nakamura et al. (2006). Evidence of magnetic reconnection in the vortices was found by Nykyri et al. (2006), matching observations with 2-D MHD simulations. Nishino et al. (2007) analysed the ions and electrons distribution functions of a $\mathrm{KH}$ event and also interpreted their observations in terms of reconnection. Hasegawa et al. (2009) identified reconnection signatures in a current sheet in the vortices.

The present study analyses in detail a series of passes through KH vortices by Cluster on the flank of the MP, behind the dusk terminator, after over $10 \mathrm{~h}$ of northward IMF. A particular sequence of ions and electrons kinetic signatures is detected repeatedly in the vortices and the present study is aimed at providing a global interpretation of the observations.

The paper is organized as follows. Section 2 describes the Cluster data set. In Sect. 3 an overview of the whole event is presented. Section 4 describes the plasma characteristics and the magnetic field topology during the passes through the vortices. The kinetic signatures within the vortices and transport across the MP are presented in Sects. 5 and 6, respectively. In Sect. 7 possible interpretations of the observations are discussed and a global scenario which accounts for all the observational aspects is presented. Finally the conclusions are reported in Sect. 8.

\section{Data set}

The event under study occurred on 25 November 2001 and the plasma ion data are provided by the Cluster Ion Spectrometer (CIS) onboard the Cluster spacecraft. The CIS experiment consists of two instruments: CODIF, which gives the three-dimensional distribution functions in the energy range 20-38000 eV for four ion species: $\mathrm{H}^{+}, \mathrm{He}^{+}, \mathrm{He}^{++}$, and $\mathrm{O}^{+}$, and $\mathrm{HIA}$, which provides the three-dimensional ions distribution functions in the energy range $5-32000 \mathrm{eV}$, with no mass separation. In this study moments and threedimensional ions distribution functions by CODIF and HIA were used. Electron data were acquired by the Plasma Electron and Current Experiment (PEACE). Each PEACE package consists of two sensors, HEEA (High Energy Electron Analyzer) and LEEA (Low Energy Electron Analyzer), mounted on diametrically opposite sides of the spacecraft.
They are designed to measure the 3-D velocity distributions of electrons in the range of $0.6 \mathrm{eV}$ to $26 \mathrm{keV}$, with a time resolution of $4 \mathrm{~s}$. The spin averaged magnetic field data from the FGM experiment onboard the Cluster spacecraft have also been used. The CIS, FGM and PEACE experiments have been described by Rème et al. (2001), Balogh et al. (2001) and Johnstone et al. (1997), respectively.

\section{Event overview}

On 25 November 2001, the Cluster spacecraft, separated by $1000-2000 \mathrm{~km}$, are skimming the dusk equatorial flank of the MP in their outbound MP crossing, and their orbit lies roughly along a meridian at around 19:00 LT and is directed southward.

Figure 1 is an overview of the whole event. Plasma and magnetic field data from ACE, located at the L1 libration point, are also reported (ACE data have been shifted to account for the convection time to Cluster's location). From the top are the ion density, bulk speed and temperature at Cluster $\mathrm{C} 1$, the solar wind dynamic pressure measured at ACE, ACE's magnetic field components, the field magnitude and components at Cluster C1. ACE's data are also plotted as red lines in the speed and field magnitude panels. The magnetic field vectors are in the GSE coordinate system. At the time of Cluster's observations the plasma is recovering after the arrival of a strong perturbation: the IMF has been steadily northward/sunward since $10 \mathrm{~h}$ and the solar wind speed always above $700 \mathrm{~km} / \mathrm{s}$. Both these conditions are particularly favourable to the onset of the Kelvin-Helmholtz instability. Note that only data from Cluster's spacecraft $\mathrm{C} 1$ are reported, as in the interval of interest the plasma data are very similar at all spacecraft.

In the earlier part of the event, Cluster has multiple MP crossings, then, starting at 03:30 UT (until 13:10 UT) it stays in the magnetosheath except for two passages in the magnetosphere (between 08:30-09:00 UT and 11:07-13:07 UT), induced by variations of the solar wind dynamic pressure.

Throughout the event, all the plasma parameters have periodic fluctuations with period of several minutes. But while these fluctuations are quasi-sinusoidal in the intervals 03:3005:30 UT and 07:00-08:00 UT in the magnetosheath, and 12:00-13:00 UT in the magnetosphere, in the earlier part of the event (i.e. before 03:30 UT) the character of the fluctuations is completely different, as will be described in the following section. This earlier interval is the object of the present study. Note that these fluctuations are absent in the simultaneous measurements by ACE and by Geotail (not shown) located upstream of the bow shock.

\section{Description of the passes through the vortices}

Figure 2a presents in detail the interval 01:50-03:30 UT. From the top are reported the ion density, bulk velocity 


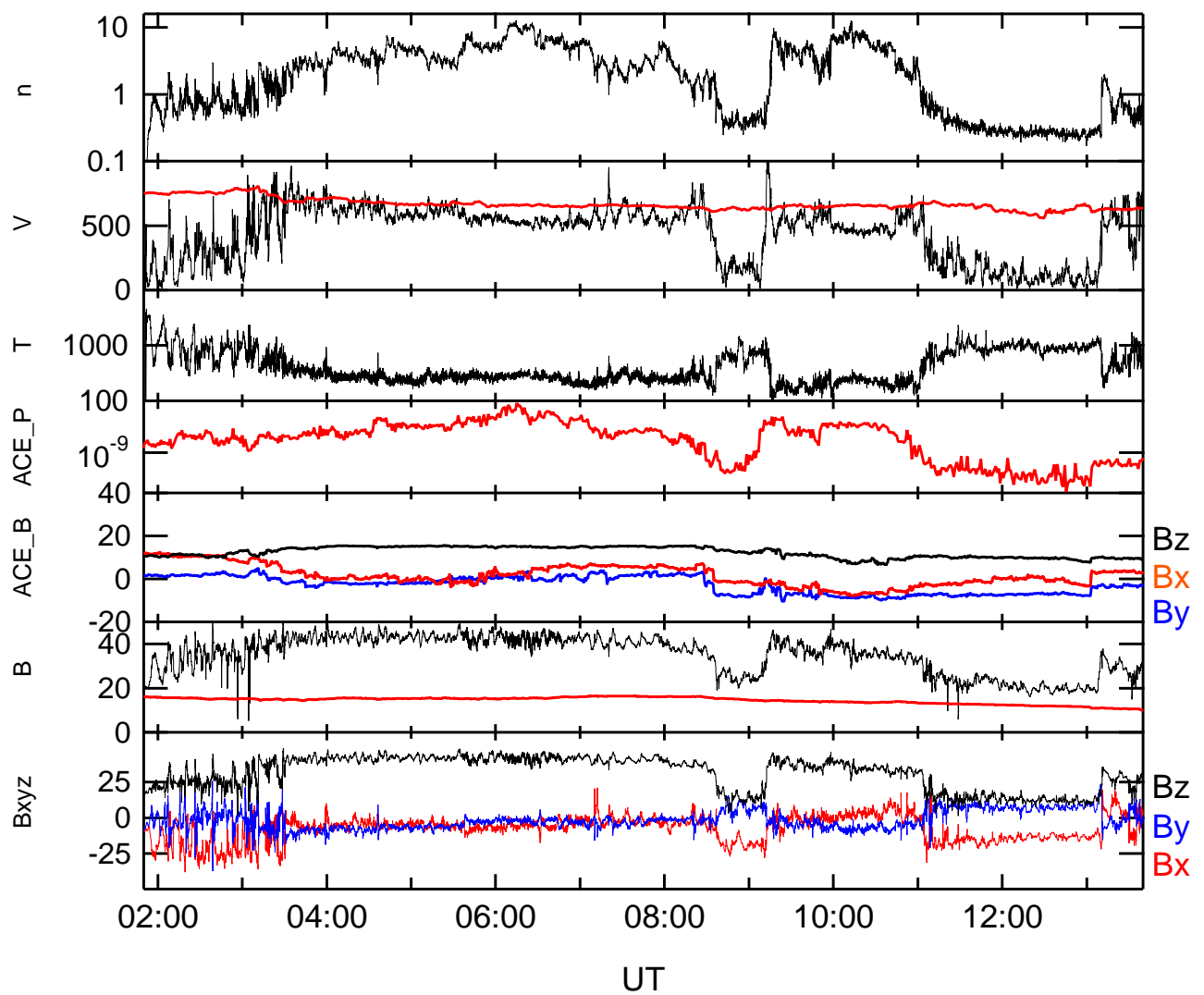

Fig. 1. Overview of the event. Data are from Cluster C1 and from ACE spacecraft (shifted to account for the convection time to Cluster's location). Plasma data on $\mathrm{C} 1$ are from CIS-HIA. From the top: the ion number density (in $\mathrm{cm}^{-3}$ ), the bulk speed (in $\mathrm{km} / \mathrm{s}$ ), the ion temperature (in $\mathrm{eV}$ ), the solar wind dynamic pressure from ACE (in $\mathrm{nPa}$ ), the magnetic field components in the GSE coordinate system and the field magnitude at ACE and at $\mathrm{C} 1$ ( $B_{\mathrm{X}}$ red, $B_{\mathrm{y}}$ blue, $B_{\mathrm{Z}}$ black) (in nT). The red line in the bulk speed panel is the solar wind speed from ACE.

components, ion temperature, magnetic field components and magnitude measured by $\mathrm{C} 1$. Vector components are in the GSE coordinate system. A periodicity of 5-10 min in all the panels is evident. Intervals with large $n, V, B$ and small $T$ occur alternately with others with small $n, V, B$ and large $T$, suggesting quasi periodic magnetopause crossings. The magnetic field components however deserve a more detailed analysis. Although they also have a recurrent behaviour, they do not simply switch between the typical magnetosheath and magnetosphere orientations, but they have a strong variability in the $B_{\mathrm{x}}, B_{\mathrm{y}}$ plane. In particular there is a strong rotation of the magnetic field projection on the $B_{\mathrm{x}}, B_{\mathrm{y}}$ plane, evidenced by the vertical lines in Fig. 2a. At the vertical lines $B_{\mathrm{y}}$ becomes negative while $B_{\mathrm{x}}$ switches to $0 \mathrm{nT}$, and later $B_{\mathrm{y}}$ smoothly returns to $0 \mathrm{nT}$ while $B_{\mathrm{x}}$ returns to negative values. These signatures are very similar to those described by Fairfield et al. (2000) (see e.g. their Fig. 5b) in their passes through $\mathrm{KH}$ vortices and to the corresponding simulations by Otto and Fairfield (2000). At 03:30 UT Cluster stops crossing the vortices and exits in the magnetosheath.
Figure $2 b$ is a zoom of a single cycle (between 02:1702:27 UT). Several regions (evidenced by the grey shadings) can be defined within the vortices, on the basis of the magnetic field characteristics and of the ion moments. Region A is the first part of the field rotation and corresponds to a decrease of $B_{\mathrm{z}}$, an increase in $B$, and to intermediate values of $n, V, T$. The second part of the field rotation is accompanied by an increase of $B_{\mathrm{z}}$ towards its maximum, while $n$ and $V$ increase and $T$ decreases slightly: this is region labelled $\mathrm{B}$. Region $\mathrm{C}$ is the part of region $\mathrm{B}$ closer to the magnetosheath, from which it differs only for the ions distribution function (Sect. 5). Next is a cold dense magnetosheath-like plasma: now $B_{\mathrm{z}}$ is maximum, the field magnitude is still maximum, and $\boldsymbol{B}$ is directed northward-dawnward (region D). Region $\mathrm{E}$ starts when the field rotates to almost northward, so that the field projection on the xy plane is minimum while $n$ and $V$ decrease. Then, while $B_{\mathrm{Z}}$ decreases toward a local minimum, the field projection on the xy plane increases and slowly rotates tailward and is usually stable, so that the field has a large negative $B_{\mathrm{X}}$, a comparable positive $B_{\mathrm{Z}}$ and a negligible 


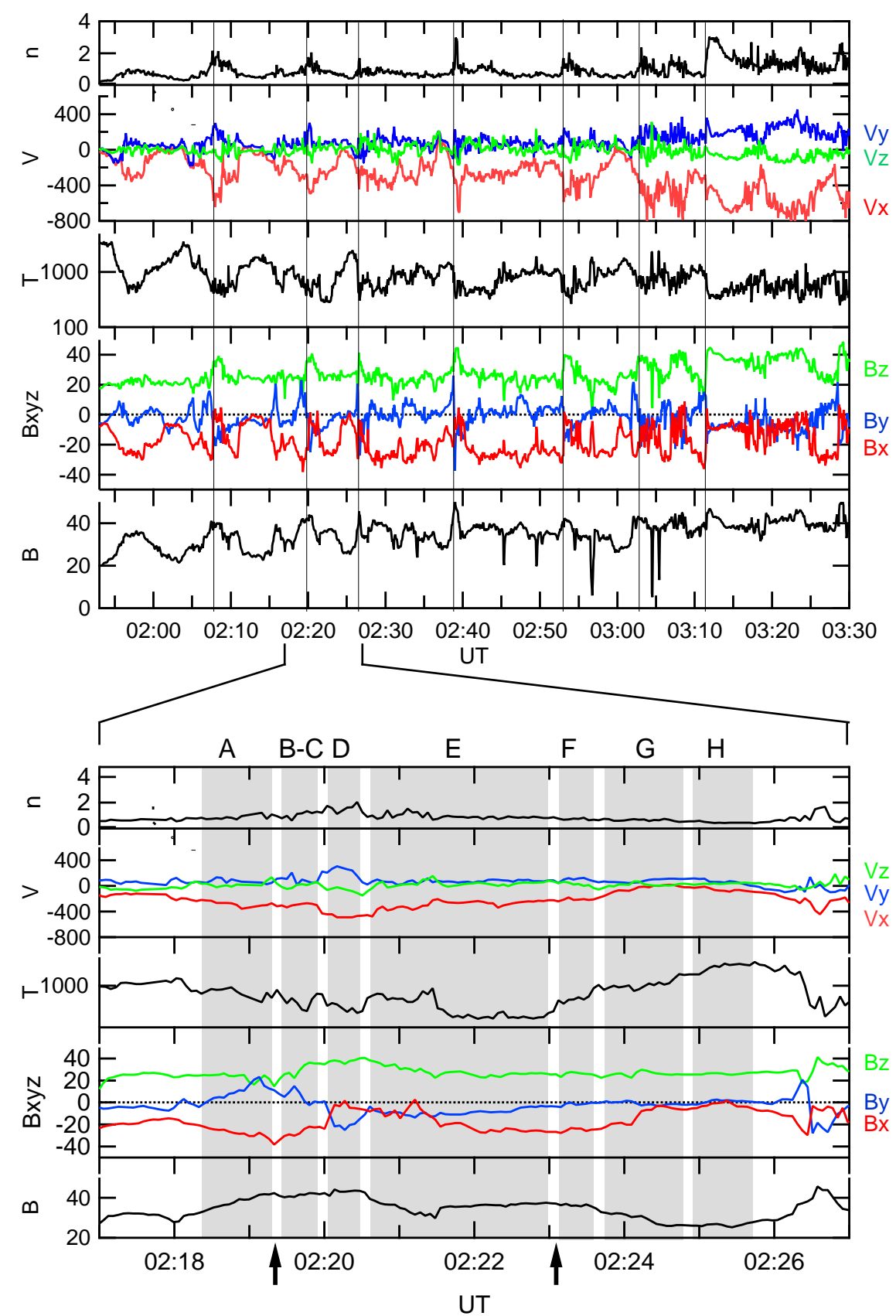

Fig. 2. Top: Overview of the passes through the vortices. Plasma data are from CIS-HIA. From the top are: the number density, the velocity vector, the ion temperature, the magnetic field and the field magnitude. Vectors are in the GSE coordinate system. Units are the same as in Fig. 1. The vertical lines mark the abrupt field rotation on the $B_{\mathrm{x}}, B_{\mathrm{y}}$ plane. Bottom: zoom on the pass across a single vortex (interval: 02:17-02:27 UT), same panels as in panel (a). The coloured shadings indicate the various regions defined in the text. The two arrows in the bottom indicate the outbound (at 02:19:21 UT) and inbound (at 02:22:59 UT) MP crossings, as identified by the ions distribution functions (Sect. 5).

$B_{\mathrm{y}}$. Note that in this particular vortex in the second half of region $\mathrm{E}(02: 21: 34-02: 23: 00 \mathrm{UT})$ is a consistent temperature decrease, lasting a couple of minutes, presumably of external origin. The end of region $\mathrm{E}$ is identified by a tempera- ture increase, which usually occurs in two steps: we call $\mathrm{F}$ and $\mathrm{G}$ the regions of magnetospheric-like plasma occurring after the first and the second temperature increase. At this point, in some of the cycles Cluster goes in a magnetospheric 


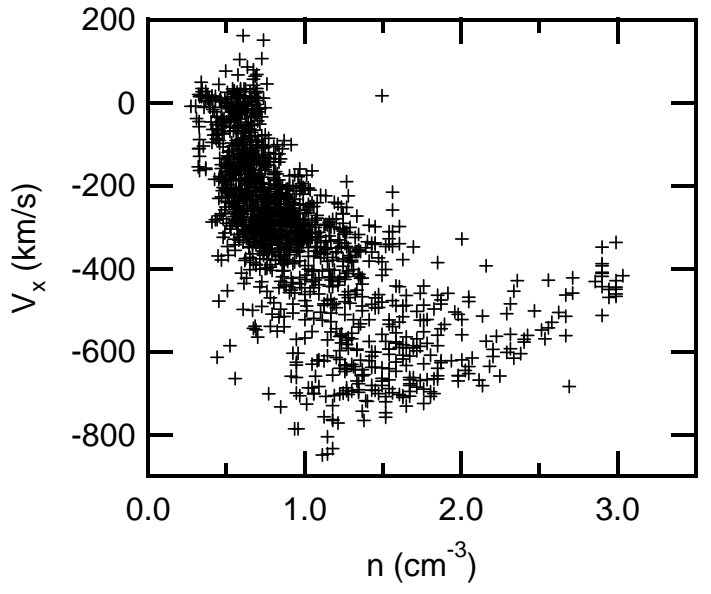

Fig. 3. Scatter plot of the ion tailward speed versus density during Cluster's passages through the vortices (time interval 01:5503:07 UT).

region (region $\mathrm{H}$ ) of hot stagnant plasma which extends to high energies, where $T$ increases further and reaches an absolute maximum (above $2000 \mathrm{eV}$ ) and $B_{\mathrm{x}}$ and $B_{\mathrm{y}}$ are usually negligible, so that the field is directed almost northward. The two black arrows at the bottom of Fig. 2b mark the outbound (at 02:19:21 UT) and inbound (at 02:22:59 UT) MP crossings: as will be clear in Sect. 5, they are better identified by changes in the ions distribution functions than by the plasma moments.

Before presenting the possible topology of the vortices, it is interesting to determine their tailward speed. Following Hasegawa et al. (2004), one can average the bulk velocity vectors during the passages through the vortices, i.e. in the time interval 01:55-03:00 UT. The result is a speed of $215 \mathrm{~km} / \mathrm{s}$ directed mainly tailward $(-207,57,7 \mathrm{~km} / \mathrm{s}$ in GSE coordinates). It is a very large speed, and taking into account the average periodicity of 5 to $10 \mathrm{~min}$, the corresponding wavelength varies between 10 and $20 R_{\mathrm{E}}$. The large size of the vortices on the one hand explains the similarity of the observations at the Cluster spacecraft, separated by only $1000-2000 \mathrm{~km}$, and on the other hand has important implications in the vortex formation, as it indicates that the vortices are generated farther upstream of the observation point and that, as pointed out e.g. by Belmont and Chanteur (1989) and by Miura (1999), they have grown to the large size observed by Cluster while propagating tailward.

Figure 3 is a scatter plot of the ion tailward speed versus density during Cluster's passages through the vortices. It clearly shows the non-monotonic relationship between density and $V_{x}$ and this, as pointed out by Hasegawa et al. (2004 and 2006) and by Takagi et al. (2006), evidences that these vortices are rolled-up, so that there are fractions of tenuous plasma faster than dense sheath plasma.

\subsection{Possible topology of the vortices}

The evolution of the magnetic field and ion parameters across the vortices in the present event is similar to the one described by Fairfield et al. (2000) (see their Fig. 5b) and in the corresponding simulation by Otto and Fairfield (2000) (see their Sect. 3.3). As a sketch of the possible magnetic field topology of the vortices in the xy plane it is therefore natural to adapt, in Fig. 4, the one proposed by Otto and Fairfield (2000). The out of plane oscillating $B_{\mathrm{Z}}$ component, which usually dominates in the passage through the vortices, is ignored in this sketch. The thick line is the magnetopause, and the shaded regions are on the magnetospheric side. The vortices, in their tailward motion, pass over the spacecraft: letters $\mathrm{A}, \ldots . ., \mathrm{H}$ correspond to the regions defined above, and indicate the regions successively explored by Cluster, roughly tracking Cluster's trajectory across the vortex. As is clear from the descriptions of Sect. 4, regions A, F, G, H are on the magnetospheric side of the vortices, and regions $\mathrm{B}, \mathrm{C}, \mathrm{E}$ on the magnetosheath side, region $\mathrm{D}$ being the magnetosheath proper. The outbound MP crossing is the passage of the trailing part of the vortex. The pass through the magnetosheath occurs where the sheath field is distorted between adjacent vortices, as indicated by the field rotation between regions B and $\mathrm{E}$. The inbound MP crossing is the passage of the leading part of the vortex over the spacecraft: later the spacecraft encounters regions $\mathrm{F}$ and $\mathrm{G}$ on the outer edge of the vortex, where the magnetic field projection on the xy plane has a stable tailward direction.

Note that, as the inspection of Fig. 2a shows, in the passages through the vortices the $\boldsymbol{B}$ vector is completely distorted with respect to its magnetosheath orientation: it is deformed and stretched by the vortex flow and acquires, on the magnetospheric side, a large negative $B_{\mathrm{x}}$ component comparable to $B_{\mathrm{z}}$, in agreement with numerical simulations (e.g. Takagi et al., 2006).

\section{Ions, electrons and oxygen kinetic signatures in the vortices}

In Sect. 4 the various regions encountered by Cluster in its passes through the vortices have been defined according to the ion moments and magnetic field data. In the present section the corresponding evolution of the ions and electrons distribution functions is presented. Eight representative examples of distributions observed in the vortices regions are shown in Fig. 5. The ions data are provided by HIA onboard $\mathrm{C} 1$ and are represented in the $V_{\|}-V_{\perp}$ plane, $V_{\|}$being the vertical axis. The electrons data are shown as 1-D cut of the distribution in phase space density units. Cuts are in the parallel (black), perpendicular (green), and antiparallel (red) directions. On top of each panel is the vortex region, as defined in Sect. 4. 


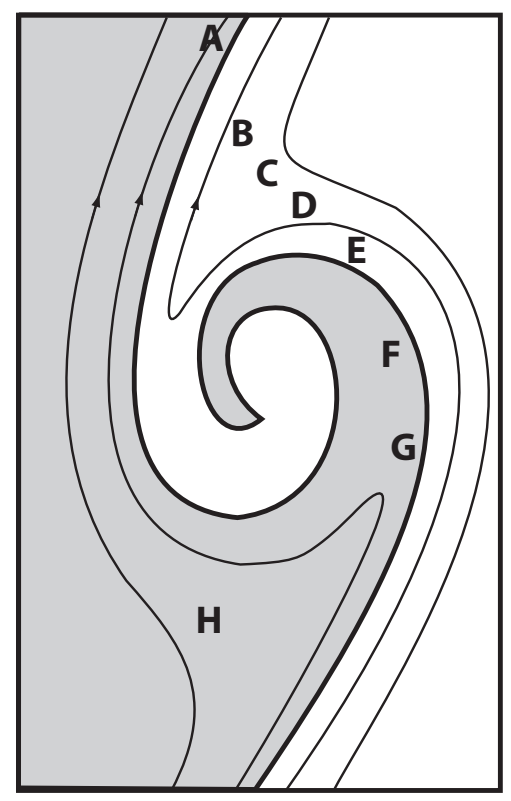

Fig. 4. Sketch of a possible topology of the vortices on the xy plane (adapted from Otto and Fairfield, 2000). The vertical axis is the $\mathrm{x}$-axis, the geomagnetic tail being upwards. The thick line is the MP, and the grey shading indicates the magnetospheric side of the vortex. Thin lines are the magnetic field lines. Letters A, B, ..., H refer to the corresponding regions defined in Sect. 4, so that the sequence $\mathrm{A}, \ldots, \mathrm{H}$ gives a rough indication of Cluster's trajectory across the vortices.

In order to describe the evolution of the distribution functions in the vortices it is easier to start from the magnetosheath and move toward the magnetosphere, rather than to follow the temporal sequence of the panels. Panels 2 through 5 have magnetosheath-like plasma and are taken in the passage of Cluster between two successive vortices in regions $\mathrm{B}, \mathrm{C}, \mathrm{D}, \mathrm{E}$ as evidenced by the cool dense ion component. Panel 4 (region D) is in the magnetosheath and has cold, dense ions and electrons populations. Panels 2, 3 (note that, due to a data gap, in panel 3 the time of the electrons is the closest time to the ion data) are in regions $\mathrm{B}$ and $\mathrm{C}$ and panel 5 in region $\mathrm{E}$. The electrons distribution functions are similar in all cases (this similarity is an important point which will be discussed in Sect. 7): they have unbalanced bi-directional flow, the parallel (antiparallel) flow dominating above (below) $200 \mathrm{eV}$. Comparing to the magnetosheath (panel 4), they are considerably heated in the parallel direction. Ions in panels 2 and 5 are similar: they consist of a cool dense low energy component together with a secondary fieldaligned heated parallel flowing component. Importantly, this secondary component is also of magnetosheath origin, as evidenced by the similarity of its thermal anisotropy, $T_{\perp}>T_{\|}$, typical of the magnetosheath (Crooker et al., 1976), with that of the main population. Unlike the electrons, the distribution of panel 3 (region C) is somewhat different: besides a cold magnetosheath plasma, it has a parallel flowing component with a low energy cutoff at speed of $\sim 580 \mathrm{~km} / \mathrm{s}$. This differs from the electron distribution function in which the parallel component varies smoothly at all energies with no evidence of a low energy cutoff. In panel 6 (at 02:22:23 UT) is another distribution function of region $\mathrm{E}$ : the ions distribution function is similar to the previous ones, but much colder, due to the temperature decrease presumably of external origin mentioned in Sect. 4. The corresponding electrons distribution function is unbalanced bi-directional with stronger flux in the parallel direction.

The detailed analysis of the complete sequence of the ions distributions (not shown) allows to identify the outbound and inbound MP crossings at 02:19:21 and 02:22:59 UT, respectively. The distribution in panel 1 (region A) is on the magnetospheric side of the outbound MP. The ions distribution consists of a parallel flow similar to the one of panel 2, but further heated, plus an antiparallel, weaker flow with a low energy cutoff at $\sim 200 \mathrm{~km} / \mathrm{s}$ and an almost isotropic high energy component. Inside the inbound MP in region F (panel 7), the ion low energy parallel flow is remarkably similar to the one outside the MP (region E, panel 6), but is further heated, and in addition there is an antiparallel heated flow with a low energy cutoff at around $250 \mathrm{~km} / \mathrm{s}$ and a quasi-isotropic high energy component. The electrons distribution functions in regions $\mathrm{A}$ and $\mathrm{F}$ have bi-directional balanced flow, with no evidence of a low energy cutoff. The ions distribution functions in regions $\mathrm{F}$ and $\mathrm{A}$ are similar: they are both in the LLBL in a symmetric position with respect to the MP: A is inside the outbound MP, and F inside the inbound MP. The similarity between regions $\mathrm{A}$ and $\mathrm{F}$ (as well as that between regions $\mathrm{B}$ and E) will be further discussed in Sect. 7. Note that in the ions distribution functions (not shown) immediately following the one in region $\mathrm{F}$, the low energy cutoff progressively decreases and further heating occurs until even the ions distribution functions become almost isotropic at high energies in region $\mathrm{G}$ (panel 8).

Finally region $\mathrm{H}$ (Fig. 6) is a passage in the magnetosphere, presumably the plasma sheet, as will be discussed in the following sections: the ion temperature increases further and the distribution function is fully isotropic and reaches high energies and the electrons are bidirectional and further heated with respect to region $\mathrm{G}$.

In Fig. 7 the profiles along $\boldsymbol{B}$ of some of the ions distribution functions are compared. In panel (a) regions $\mathrm{E}$ and $\mathrm{F}$ are compared (on opposite sides of the inbound MP): as reported previously, at low energy the fluxes are almost identical, evidencing a common origin (apart from the low energy cutoff of region $\mathrm{F}$ in the antiparallel direction) but at high energy region $\mathrm{F}$ is almost isotropic. The next two panels compare regions on the magnetospheric side of the MP. In panel (b) regions $\mathrm{F}$ and $\mathrm{G}$ are compared: apart from the low energy cutoff of region $\mathrm{F}$, hardly visible in region $\mathrm{G}$, the two profiles are similar but in $\mathrm{G}$ is further heating. Finally in panel (c) 

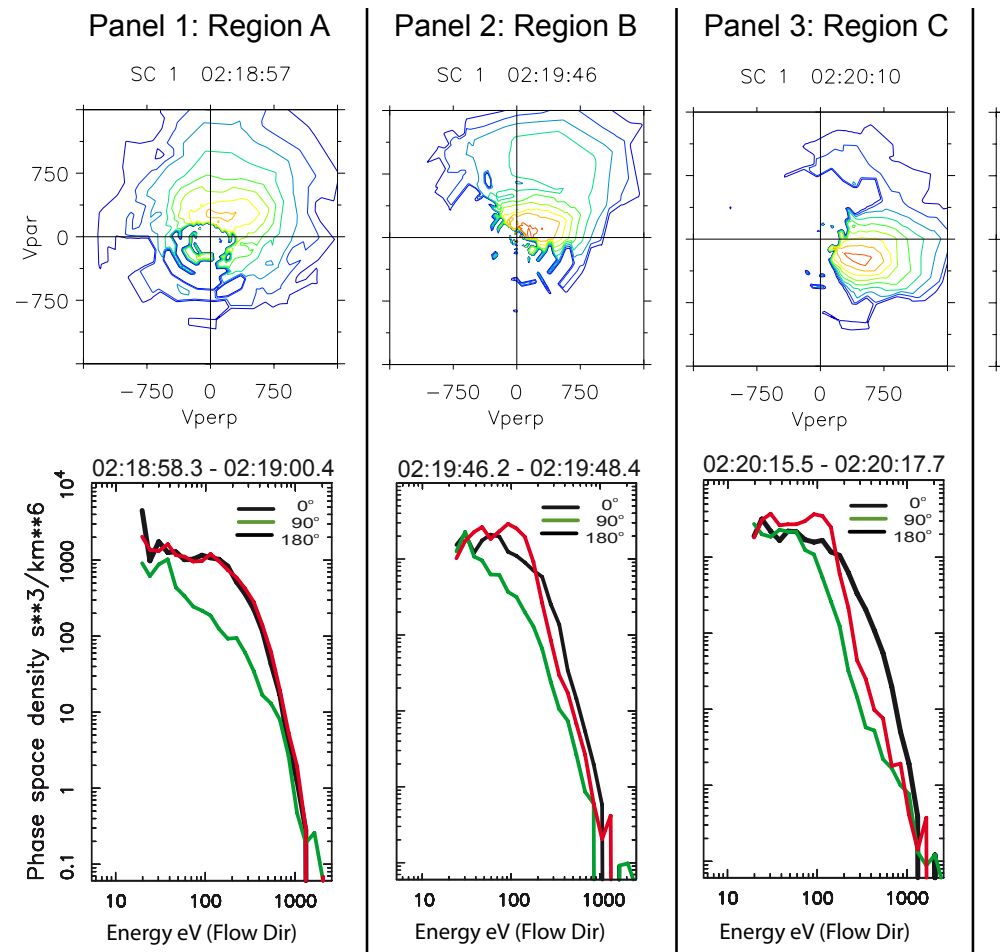

Panel 4: Region D
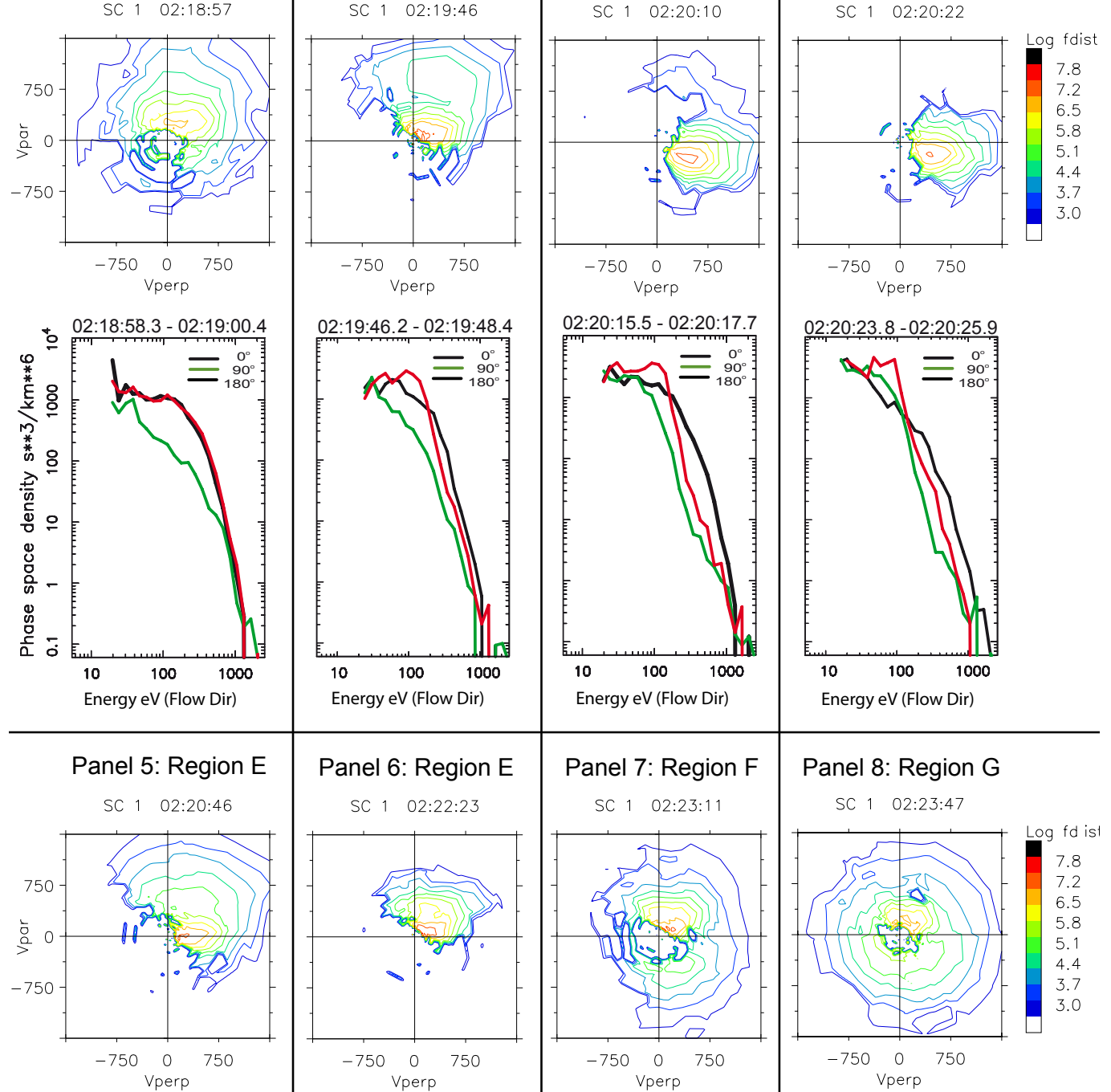

Panel 6: Region E

SC $102: 22: 23$
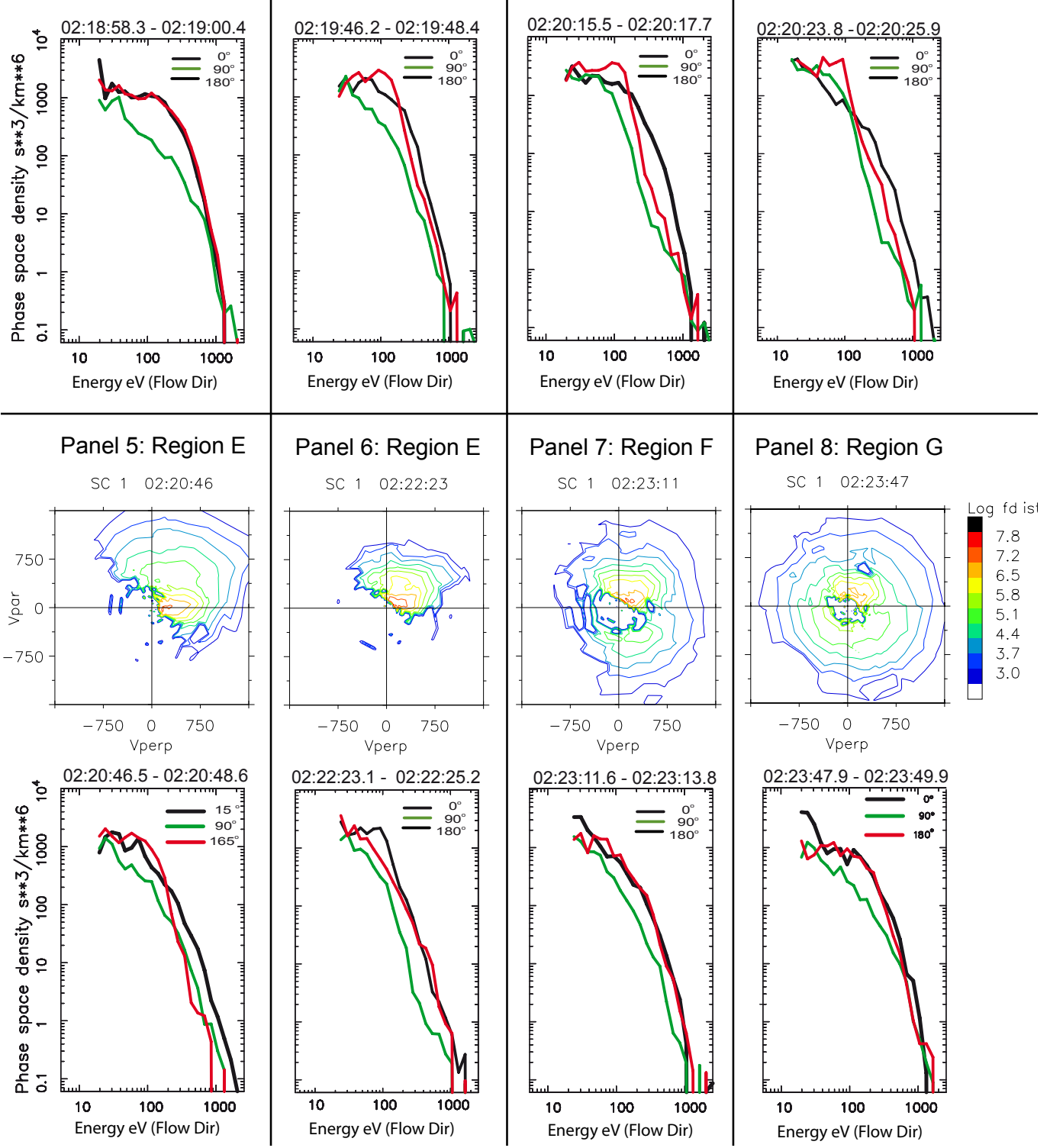

Panel 8: Region G

SC 1 02:23:47
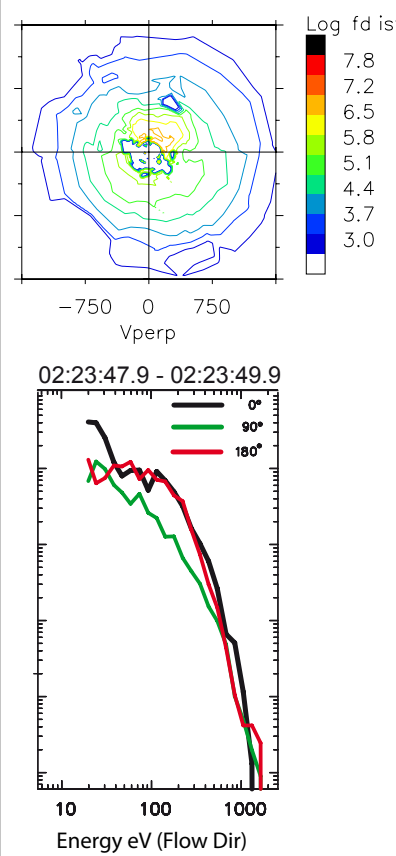

Fig. 5. Evolution of the ions and electrons distribution functions (measured by CIS-HIA and PEACE respectively) across the vortices. The figure is separated in eight panels, each displaying an ion and an electron distribution function. On top of each panel is the corresponding vortex region, as defined in Sect. 4. Ions distribution functions are represented in the $V_{\|}-V_{\perp}$ plane, the phase space density is expressed in $\mathrm{km}^{-6} \mathrm{~s}^{3}$, and the velocity in $\mathrm{km} / \mathrm{s}$. The electrons distribution functions are shown as 1-D cut of the distribution in phase space density units. Cuts are in the parallel (black), perpendicular (green), and antiparallel (red) directions. 


\section{Region $\mathrm{H}$}
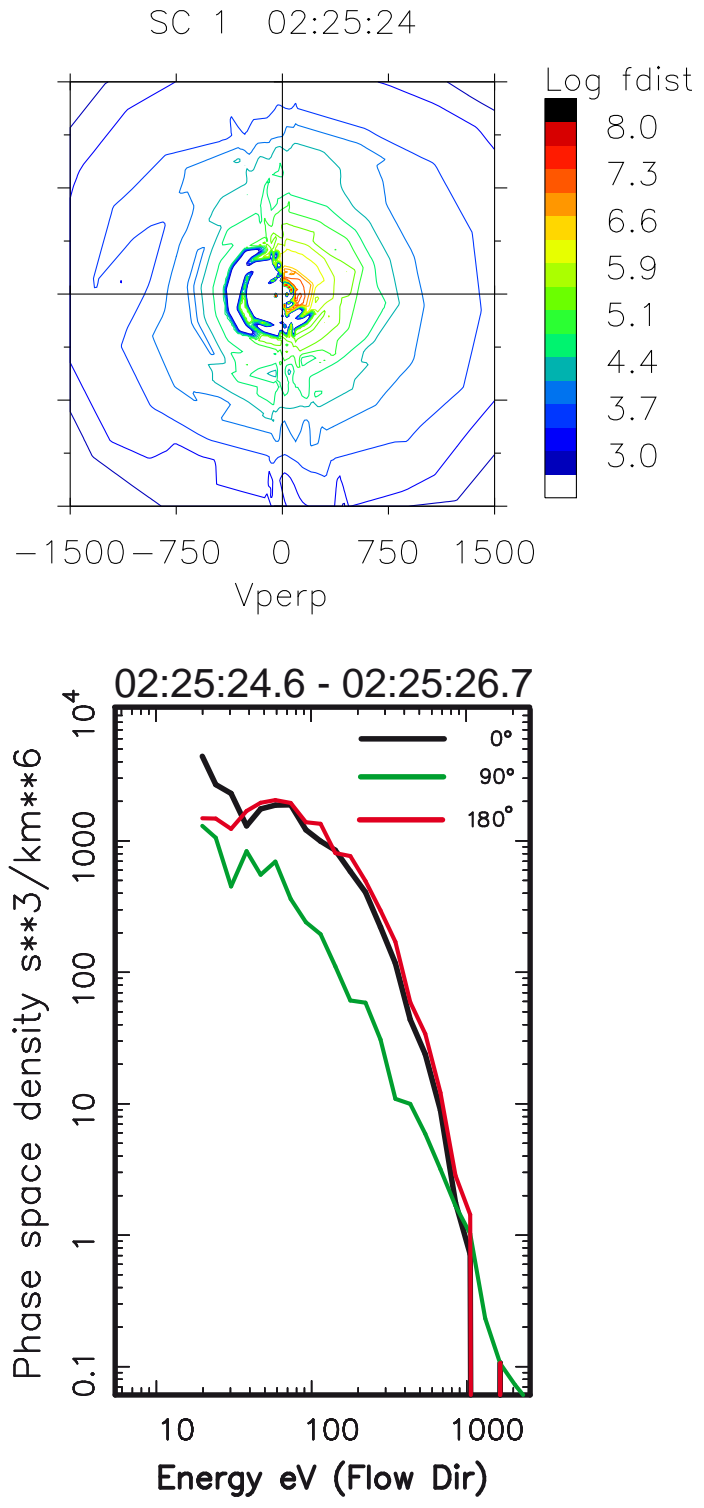

Fig. 6. Ions and electrons distribution functions for region $\mathrm{H}$, defined in Sect. 4. Same format as Fig. 5.

regions $\mathrm{G}$ and $\mathrm{H}$ are compared: in region $\mathrm{H}$ are equal heated counterstreaming fluxes, but the two profiles differ at all energies, indicating a different origin (i.e. magnetospheric origin in region $\mathrm{H})$. The important point which emerges from Figs. 7 and 5 is the gradual evolution of the ions distribution functions through regions $\mathrm{E}, \mathrm{F}$, and $\mathrm{G}$. This point will be further discussed in Sect. 7.

The sequence of distribution functions of Figs. 5 and 6 is typical of the passes through the vortices of the present event, although not all passes have the complete set, due to the fast crossing of some of the vortex regions. On the other
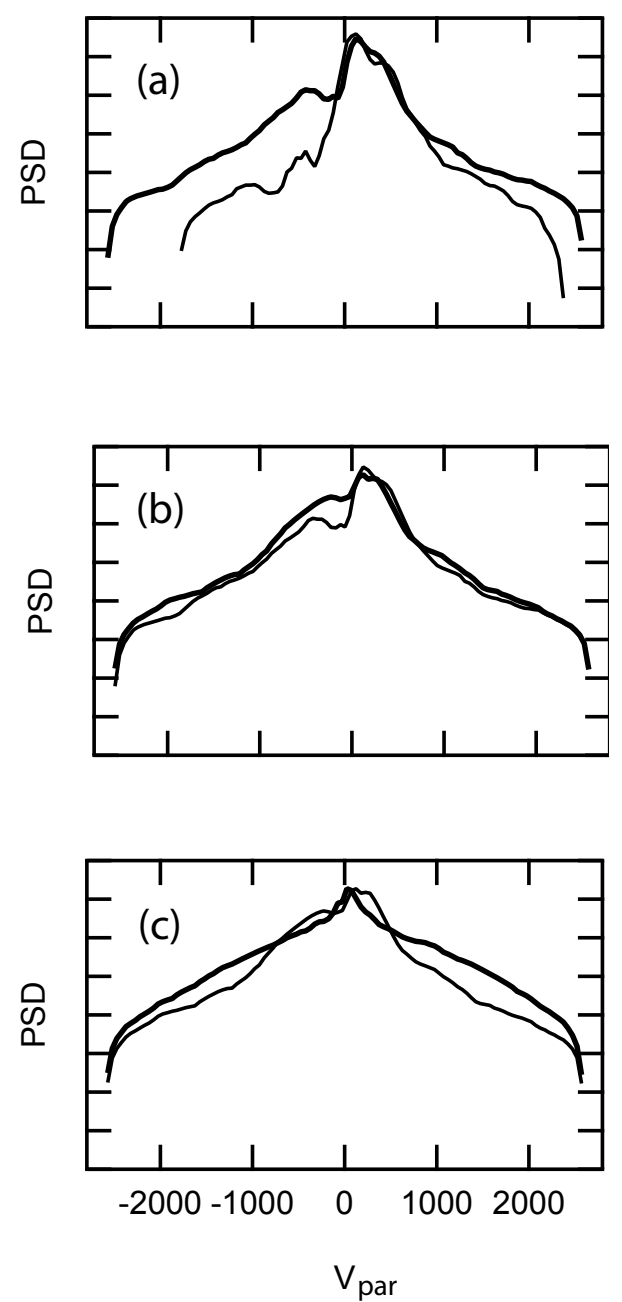

Fig. 7. Comparison between the profiles along $\boldsymbol{B}$ (in arbitrary units) of some of the distribution functions of Figs. 5 and 6: in panel (a) are regions $\mathrm{E}(02: 22: 23 \mathrm{UT})$ (thin line) and $\mathrm{F}(02: 23: 11 \mathrm{UT})$ (thick line), in panel (b) regions $F(02: 23: 11 \mathrm{UT})$ (thin line) and $\mathrm{G}$ (02:23:47 UT) (thick line), and in panel (c) regions G (02:23:47 UT) (thin line) and H (02:25:24 UT) (thick line).

hand, there are vortices in which the passage from the magnetosheath (region D) to the inbound or to the outbound MP is slower than in the case of Fig. 5, and in that case the parallel low energy cutoff gradually decreases as the MP is approached.

In Table 1 the observations of the various regions are summarized. Column 2 indicates the regions' properties based on the magnetic field and on the ions moments. Columns 3 and 4 report the ions and electrons kinetic properties (Sect. 5) and column 4 refers to the reconnection process which will be discussed in Sect. 7.

One important point which emerges from the observations described above is the presence of multiple field-aligned ion components in the regions which surround the outbound and 
Table 1. A brief summary of the regions' properties presented in Sects. 4 and 5. In column 1 is the region; in column 2 are its properties as derived from $\boldsymbol{B}$ and from the moments; in columns 3 and 4 are the ions and electrons kinetic features (Sect. 5); and the last column refers to the reconnection scenario proposed in Sect. 7. Legend: APF: antiparallel flow; HE: high energy; LE: low energy; PF: parallel flow; q-isotropic: quasi-isotropic; $T$ : temperature.

\begin{tabular}{|c|c|c|c|c|}
\hline Region & $\boldsymbol{B}$ and ion moments & Ions distrib. funct. & Electrons distrib. funct. & Role in reconnection \\
\hline A & $\begin{array}{l}\text { first part of } \boldsymbol{B} \\
\quad \text { rotation }\end{array}$ & $\begin{array}{c}\text { heated PF, APF cutoff, } \\
\text { HE q-isotropic }\end{array}$ & balanced & $\begin{array}{l}\text { outbound } \\
\text { LLBL }\end{array}$ \\
\hline B & $\begin{array}{l}\text { 2nd part of } \boldsymbol{B} \\
\text { rotation }\end{array}$ & $\begin{array}{c}\text { cold at low energy, } \\
\text { heated PF }\end{array}$ & $\begin{array}{c}\text { unbalanced } \\
\text { bi-directional }\end{array}$ & $\begin{array}{l}\text { inner outbound } \\
\text { MSBL }\end{array}$ \\
\hline $\mathrm{C}$ & $\begin{array}{l}\text { adjacent to } \\
\text { magnetosheath }\end{array}$ & $\begin{array}{l}\text { cold at low energy } \\
\text { and cutoff in PF }\end{array}$ & $\begin{array}{c}\text { unbalanced } \\
\text { bi-directional }\end{array}$ & $\begin{array}{l}\text { outer outbound } \\
\text { MSBL }\end{array}$ \\
\hline $\mathrm{D}$ & $\mathrm{T} \min$ & cold & $\begin{array}{c}\text { cold } \\
\text { unidirectional flow }\end{array}$ & magnetosheath \\
\hline $\mathrm{E}$ & small $B_{\mathrm{y}}$ & $\begin{array}{c}\text { cold at LE } \\
\text { and heated PF }\end{array}$ & $\begin{array}{c}\text { unbalanced } \\
\text { bi-directional }\end{array}$ & $\begin{array}{l}\text { inner inbound } \\
\text { MSBL }\end{array}$ \\
\hline $\mathrm{F}$ & 1st $T$ increase & $\begin{array}{c}\text { heated PF, APF cutoff } \\
\text { HE q-isotropic }\end{array}$ & balanced & outer LLBL \\
\hline G & 2nd $T$ increase & q-isotropic & balanced & $\begin{array}{l}\text { inner } \\
\text { LLBL }\end{array}$ \\
\hline $\mathrm{H}$ & $T$ max, $\boldsymbol{B}$ north & hot q-isotropic & balanced & magnetosphere \\
\hline
\end{tabular}

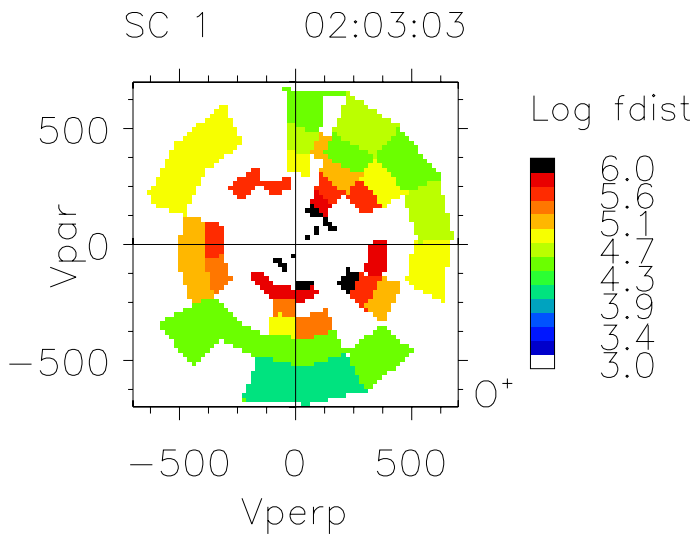

Fig. 8. Phase space density of $\mathrm{O}^{+}$ions (obtained by CIS-CODIF) in the $V_{\|}-V_{\perp}$ plane, in the magnetosphere, region $\mathrm{H}$. The phase space density is expressed in $\mathrm{km}^{-6} \mathrm{~s}^{3}$, and the velocity is in $\mathrm{km} / \mathrm{s}$.

inbound MP (i.e. regions A, B, C and regions $\mathrm{E}, \mathrm{F}$ ): this finding suggests that reconnection is going on, so that whenever Cluster is close to the MP, in the current sheet, it detects field aligned ion populations. Regions B, C and E are the magnetosheath boundary layer (MSBL) and regions $\mathrm{A}$ and $\mathrm{F}$ the LLBL. The issue is therefore to understand where does reconnection occur: does it occur locally, within the vortex, as observed in other events reported in the literature (e.g. Nykyri et al., 2006; Hasegawa et al., 2009), or does it occur on a global scale, the reconnection site being far from the vortex? In Sect. 7 possible interpretations will be discussed.
Let us now consider the oxygen ions, measured by CISCODIF. In the present event $\mathrm{O}^{+}$ions are abundant in the magnetosphere where, as shown in Fig. 8, they are almost isotropic. On the contrary, on the magnetosheath side of the MP, in the MSBL, the $\mathrm{O}^{+}$fluxes are quite low and, importantly, there is no unambiguous evidence of field-aligned flow. If reconnection were occurring within the vortex, in the MSBL Cluster would detect magnetospheric transmitted $\mathrm{O}^{+}$ions, which would appear as field-aligned beams. The absence of $\mathrm{O}^{+}$ions beams in the MSBL has therefore important consequences, as it rules out the hypothesis of local reconnection.

\section{Plasma transport across the MP}

Evidence of plasma transport across $\mathrm{KH}$ vortices from the magnetosheath to the magnetospheric side of the vortices was found by Hasegawa et al. (2004). Comparing two Cluster spacecraft, they found a larger density in the most earthward spacecraft, besides observing the coexistence of magnetosheath and magnetospheric plasma on the magnetospheric side of the vortices. They attribute this transport to the effect of rolled-up vortices. In the present event the plasma transport across the MP is further evidenced by using the ion composition (measured by CODIF) as a tracer of the ion origin: $\mathrm{O}^{+}$is considered as a tracer of magnetospheric plasma, and $\mathrm{He}^{++}$of solar wind. Figure 9 shows the histograms of the time of flight of CODIF analyser separately for the low (i.e. below $2600 \mathrm{eV}$ ) and high (i.e. above $7000 \mathrm{eV}$ ) energy channels in the magnetospheric side of the vortex, region $\mathrm{G}$, in the time interval 02:47:30-02:49:33 UT). 

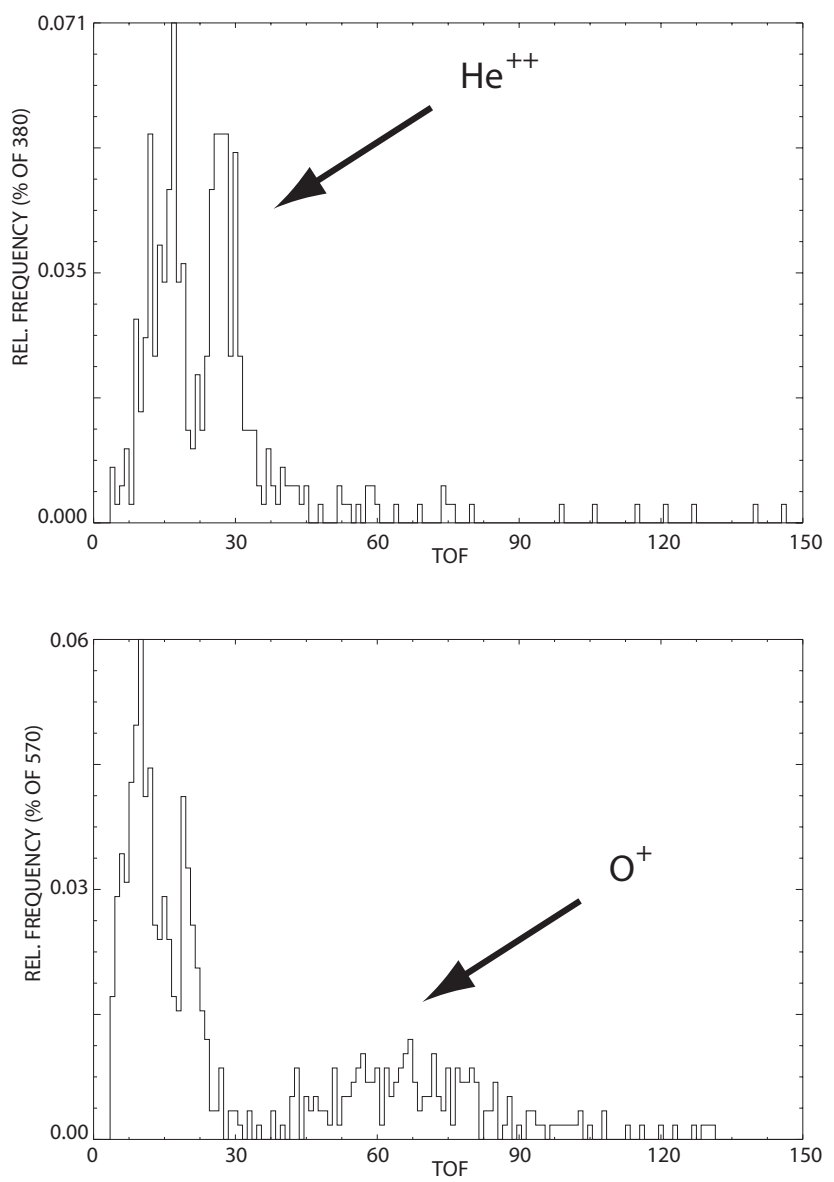

Fig. 9. Histograms of the ions time-of-flights (TOF) obtained by CIS-CODIF in region $\mathrm{G}$, on the magnetospheric side of the vortex (time interval: 02:47:30-02:49:33 UT), in two energy ranges. In the abscissa is the TOF channel number (inversely proportional to the ion velocity) and in the ordinate axis is the percent of the particle number in each TOF channel. At low energies (upper plot) is the $\mathrm{He}^{++}$peak, indicating a magnetosheath origin, and at high energy is the $\mathrm{O}^{+}$peak, indicating a magnetospheric origin.

A remarkable difference in composition in the two parts is evident: while in the high energy plot is an $\mathrm{O}^{+}$peak, below $2600 \mathrm{eV}$ is a clear alpha peak, indicating a solar wind origin for this part of the distribution functions and thus confirming the occurrence of plasma transport to the magnetospheric side of the vortex. A similar plot (not shown) made in magnetospheric region $\mathrm{H}$ does not provide an unambiguous evidence of the presence of solar wind plasma.

\section{Discussion}

The present study concerns very large (10-20 $\left.R_{\mathrm{E}}\right)$ fully developed $\mathrm{KH}$ vortices repeatedly explored by Cluster on the equatorial dusk flank of the MP, which travel tailward at a speed of $215 \mathrm{~km} / \mathrm{s}$. In the vortices is a complex sequence of ions and electrons distribution functions, described in detail in Sect. 5, which is particularly interesting and which is the focus of the present study. In the present section possible interpretations of the observations will be discussed, until a satisfactory scenario which accounts for all the observations will be proposed. Let us summarize here the main observational facts described in the previous sections:

1. the large vortices are rolled-up, and this indicates that they have been generated well upstream of the observation point and that they have grown to the observed size and have rolled-up while propagating tailward;

2. all the vortices are similar to one another, and the evolution of the distribution functions is the same in all the vortices;

3. inside each vortex, in the regions surrounding the MP, the ions distribution functions consist of multiple field aligned components. The detailed features of these components vary depending on the position in the vortex, but their presence close to the MP, i.e. in the current sheet, suggests that reconnection is going on. The main issue is to determine the reconnection topology, whether it occurs locally within the vortex or whether it occurs on a global scale;

4. the structure of the MP current layer is identical in the inbound and in the outbound MP in the vortices, in spite of its twisting;

5. there is no clear evidence of field aligned $\mathrm{O}^{+}$ions in the current sheet, while they are abundant in the nearby magnetosphere. This is an important finding which allows to exclude reconnection occurring locally between the magnetosheath and magnetospheric field;

6. magnetosheath and magnetospheric plasma coexist on the magnetospheric side of the vortices, similarly to earlier observations (Hasegawa et al., 2004).

Let us now consider possible reconnection configurations. Having excluded local reconnection (item 5 above), then reconnection should occur on a global scale. In this respect it is important to recall that, prior to Cluster's observations, the IMF had been persistently northward with positive $B_{\mathrm{x}}$, since about $10 \mathrm{~h}$. This configuration favours high latitude reconnection and in particular, considering also the winter season, favours high latitude reconnection in the Southern Hemisphere (Crooker, 1992). Let us first consider the case of poleward of the cusp reconnection in the Southern Hemisphere. This process reconnects sheath and open lobe field lines and produces one field line connected to the southern ionosphere and open in the Northern Hemisphere, together with one completely detached field line. If the reconnection process is stable (this point will be discussed below) the 
open reconnected field lines contract away from the reconnection site, then drape around the flank of the MP forming the MSBL and "sink" tailward-duskward across the MP forming the LLBL (Song and Russell, 1992). In the present event reconnected field lines would become embedded in the vortices. An observer on the ecliptic plane, as in the present event, would observe different kinetic signatures depending on whether the reconnected field line lies on the magnetosheath or on the magnetospheric side of the MP. Kinetic signatures in this reconnection topology have been studied in detail by Fuselier et al. (1995, 1997) and by Bogdanova et al. (2008) with spacecraft located near the subsolar magnetopause.

Figure 10 is a sketch of the proposed scenario which shows the evolution of the reconnected field lines across the MP in the vortices: quasi-steady reconnection poleward of the southern cusp occurs and the reconnected field lines with one foot in the southern ionosphere are initially on the sheath's side of the MP and then are progressively convected across it. Full circles on the field lines mark the field lines' encounter with the MP, and correspondingly the blue and green part of the field line are on the magnetospheric and on the sheath side of the MP respectively. As time progresses, the field line's encounter with the MP moves northward. Cluster, while passing through the vortices, goes repeatedly across the MP, and encounters the reconnected field lines. At one time Cluster is in the magnetosheath proper, then it moves toward the inbound MP, encountering successively reconnected field lines 1, 2, 3, and 4. At Cluster's location, near the equatorial plane, field lines 1 and 2 are both on the magnetosheath's side of the MP, in the MSBL, but field line 1 has been reconnected more recently than field line 2. Similarly, at Cluster's location field lines 3 and 4 have sunk in the MP and form the LLBL, field line 3 having sunk more recently than field line 4 , so that lines 3 and 4 are in the outer, inner LLBL respectively.

According to this scenario, the reconnected field lines undergo two processes, one is the sinking into the magnetosphere, which, as shown by Song and Russell (1992), occurs with the Alfvén speed along the MP, and the other is the convection around the flanks of the magnetosphere to Cluster's location. If the former time were shorter than the latter, then Cluster would detect only field lines of type 3 and 4 (i.e. the LLBL). On the contrary the observational fact that Cluster systematically detects MSBL besides LLBL field lines suggests that the convection time around the flanks of a recently reconnected field line is short compared to the "sinking" time. In order to check this hypothesis, we applied the Cooling et al. (2001) numerical model which follows the motion of the reconnected flux tube. First of all it was checked that, following the flux tube reconnected in the Southern Hemisphere which contracts northward at a position comparable to that of Cluster, the computed plasma density and speed are similar to the measured ones: this gives confidence in the model results. According to the Cooling model, in the

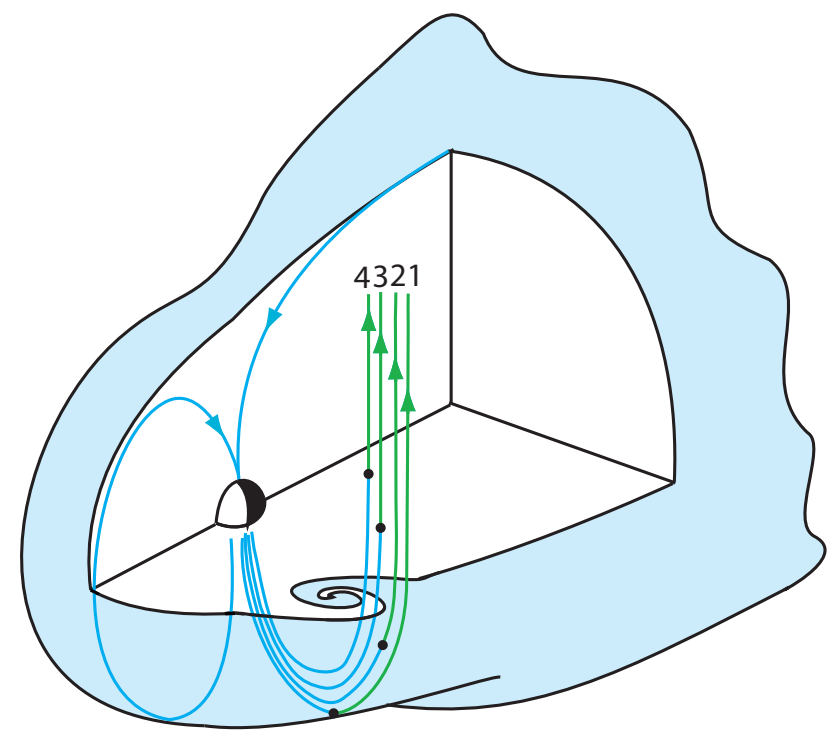

Fig. 10. Sketch of a possible scenario which accounts for the observations: lobe reconnection occurs in the Southern Hemisphere. The reconnected field line with one foot in the southern ionosphere convects tailward and duskward, becoming embedded in the vortices. As time progresses, the MSBL field line 1 (the most recently reconnected field line) is progressively convected at positions 2, 3 and 4 successively. The black circle on each field line is the intersection of the field line with the MP, so that the blue and green curves are on the magnetospheric and the magnetosheath side of the MP, respectively. At Cluster's location on the equatorial plane field lines 1 and 2 are part of the MSBL, field line 1 being more recently reconnected. Similarly, field lines 3 and 4 are part of the LLBL, field line 3 being more recently convected across the MP.

present geometry reconnection can occur along an extended $\mathrm{X}$-line at the terminator in the Southern Hemisphere. This is an important finding which strongly confirms the compatibility of the proposed scenario with the observations.

Of course this scenario relies crucially on the stability of the reconnection process, and this in turn requires that, upon reconnection, the two reconnected field lines contract in opposite directions, and in particular that the reconnected open field line contracts sunward. This can occur only if the magnetosheath's flow at the reconnection site, i.e. at the southern lobe, is subalfvénic (Cowley, 1982; Gosling et al., 1991). The lack of a local observation point does not allow to verify this fact directly. However, the Alfvén and magnetosheath speeds close to the reconnection site obtained by the Cooling et al. (2001) model are $V_{\mathrm{A}}=640 \mathrm{~km} / \mathrm{s}$ and $V=520 \mathrm{~km} / \mathrm{s}$ respectively, thus confirming that the sheath's flow is subalfvénic, so that the reconnection process can be stable.

Another issue concerns the origin of the secondary heated ion population in the MSBL (Fig. 5, panels 2 and 5), which is of magnetosheath origin as discussed in Sect. 5. One possibility would be that it consists of magnetosheath ions reflected at the reconnection site. According to Cowley (1982) 
in this case the peaks of the incident and of the reflected components should be separated by $2 \cdot V_{\mathrm{A}}$, where $V_{\mathrm{A}}$ is the Alfvén speed at the reconnection site. (Ions reflected at the reconnection site have been observed by several authors, e.g. Gosling et al., 1991; Fuselier et al., 1991; Retinò et al., 2005). Using the estimate of the Alfvén speed at the reconnection site obtained above, we see that the observed separation between the two peaks is smaller than $2 \cdot V_{\mathrm{A}}$, thus excluding the interpretation of sheath plasma reflection at the reconnection site. Therefore an alternative possibility is that the sheath ions traveled to the ionosphere, mirrored and returned to Cluster, after being heated in crossing the MP.

Let us now consider the ions and electrons kinetic signatures in the vortices. The electrons distribution functions on MSBL field lines 1 and 2 (field line 1 corresponds to the outer MSBL, i.e. region $\mathrm{C}$ and field line 2 to the inner MSBL, i.e. to regions $\mathrm{B}$ and $\mathrm{E}$ ) are similar, consisting of a low energy cold flux and of a heated parallel flow. The former is the sheath flux, while the latter is interpreted as the incoming sheath population which mirrored in the ionosphere, and returned to the spacecraft after being heated at the reconnection site. The corresponding ions distribution functions on field lines 1 and 2 are remarkably different: while on field line 2 (Fig. 5, panels 2, 5 and 6) the ions are similar to the electrons (i.e. cold magnetosheath flow at low energy and a heated parallel flow), on field line 1 (Fig. 5, panel 3) there is only the incident cold magnetosheath population, together with a high energy beam with a low energy cutoff. The reason is that, on the more recently reconnected field line (i.e. field line 1), the slower parallel moving ions did not have time to cross the MP, go to the ionosphere, mirror and go back to Cluster, so that only the high energy part of the distribution reaches Cluster. On the contrary, the fast moving electrons have plenty of time to make the whole trip, even on the more recently reconnected field line.

As shown in Fig. 10, field lines "sunk" in the MP (i.e. field lines 3 and 4) encounter the MP north of Cluster. On the recently sunk field line 3 (i.e. outer LLBL, region F), as a consequence of the sinking process, the parallel ion flow is practically identical to that of the MSBL (i.e. field line 2, region E, Fig. 5 panel 6, and Fig. 7 panel a), except for a further heating due to the MP crossing and to a progressive merging with a high energy isotropic population resident locally, in the equatorial magnetosphere. The antiparallel flow consists of sheath plasma heated where the field line encounters the MP, north of Cluster and has a low energy cutoff. On field line 4 (region G) the antiparallel cutoff disappears and further heating occurs. The electrons on the contrary have similar, counterstreaming flows both on field lines 3 and 4 (Fig. 5 panels 7 and 8). The interpretation is therefore that, on the field line which more recently crossed the MP (i.e. field line 3), only the fastest moving antiparallel ions had time to reach Cluster, as evidenced by the antiparallel low energy cutoff (Fig. 5, panel 7).
Finally region $\mathrm{H}$ (Fig. 6), characterized by the concurrent presence of isotropic $\mathrm{O}^{+}$ions (Fig. 8) and balanced ions and electrons flow (electrons are very similar to those of region G), is interpreted to lie on closed field lines: it is in the magnetosphere earthward of the vortices, presumably the plasma sheet. This is consistent with Fig. 7, panel (c), which suggested a magnetospheric origin for region $\mathrm{H}$.

In summary, the proposed scenario for the 25 November 2001 event accounts for all the observational facts. It is based on the occurrence, on a global scale, of long lasting lobe reconnection in the Southern Hemisphere, favoured by the IMF geometry and by the season. Reconnection occurs along an extended X-line and the reconnected field lines, after convection across the MP, become embedded in the vortices, so that Cluster sees reconnection signatures whenever it is in the current sheet inside the vortices. Regions B, C and E are the MSBL and regions A, F and G are the LLBL. The important consequence is that the magnetopause structure is identical in the various parts of the vortices (item 4 above). The proposed interpretation accounts for the kinetic signatures of ions and electrons; also the absence of $\mathrm{O}^{+}$ions in the current sheet can be accounted for, in the case of absence of ionospheric outflow during this event.

So far poleward of the cusp reconnection has been considered. However high latitude reconnection equatorward rather than poleward of the cusp (Fuselier et al., 1997) cannot be a priori excluded. In this case reconnection occurs between sheath field lines and closed magnetospheric field lines (resulting in two open field lines, connected to the northern/southern ionosphere) and the field lines do not sink across the MP, but ions stream along the field lines, while they contract tailward. Under this hypothesis, the heated parallel ion flow in the MSBL (regions B and E) should be interpreted as transmitted magnetospheric component, but this contrasts with its observed thermal anisotropy typical of the magnetosheath (Sect. 5). So reconnection equatorward of the cusps seems less likely than lobe reconnection.

There is one important issue regarding the electrons distribution functions: the almost balanced distribution functions observed in the magnetospheric side of the vortices in regions $\mathrm{A}$ and $\mathrm{F}$ (Fig. 5) could at first sight be interpreted as an indication that Cluster is on closed field lines. However this interpretation must be ruled out, because the corresponding ions distribution functions are not compatible with a closed topology. In analogy with Fuselier et al. $(1995,1997)$ and Bogdanova et al. (2008), the interpretation is that these field lines are open, but the electrons in the antiparallel direction are subject to heating upon crossing the MP, north of Cluster, so that they appear quasi-balanced.

As a final point let us consider the issue of plasma transport. As already reported, Hasegawa et al. (2004) observing the coexistence of solar wind and magnetospheric plasma on the magnetospheric side of the vortex, suggested that rolling up of the vortices could be responsible for the transport of solar wind plasma to the magnetospheric side. Evidence of 
plasma transport in the present event has been presented in Sect. 6, but here the rolling up of the vortices is not expected to be the only transport mechanism. Rather, in this particular event, lobe reconnection provides an efficient means of transport of magnetosheath plasma on a global scale, from high to low latitude. The rolling up of the vortices could possibly be a further transport mechanism on a local scale.

\section{Conclusions}

During a long lasting period of northward IMF and of large solar wind speed, Cluster goes across very large rolled-up $\mathrm{KH}$ vortices on the dusk equatorial MP. The large size of the vortices suggests that they were not generated locally, but farther upstream at the dayside MP and that they have successively grown and rolled-up while propagating tailward. The present study is focussed in the interpretation of a particularly interesting sequence of ions and electrons distribution functions detected in all the vortices: systematically, whenever Cluster is close to the MP, the ions distribution functions have multiple field aligned components, suggesting the occurrence of reconnection. Unlike other studies reported in the literature, the characteristics of the observed distribution functions are not compatible with local reconnection, but are fully accounted for by lobe reconnection occurring along an extended X-line in the Southern Hemisphere. The reconnected field lines, while convecting tailward and duskward, become embedded in the vortices. As Cluster travels across the vortices, it goes in and out of the current layer, and the observed kinetic signatures of the ions and electrons are in excellent agreement with this interpretation, provided the different speeds of electrons and ions are taken into account.

Another observational aspect is the transport of solar wind plasma to the magnetospheric side of the vortices, unambiguously evidenced by CODIF ion composition experiment. Also this aspect is accounted for by the proposed scenario, as transport can be due on the large scale to the reconnection process which takes the magnetosheath plasma from high to low latitude, and on the local scale possibly to the rolling up of the vortices.

Finally it must be stressed that reaching a satisfactory and coherent interpretation of the present observations has only been possible thanks to the simultaneous analysis of the detailed kinetic signatures of all the plasma components, protons, electrons and $\mathrm{O}^{+}$ions.

Acknowledgements. We thank the CDAWeb team for providing the ACE MAG (P.I.: N. F. Ness) and ACE SWEPAM (P.I.: D. J. McComas) data. The work done at IFSI has been supported by the Agenzia Spaziale Italiana, under contract ASI-INAF I/023/09/0 "Attività Scientifica per l'Analisi Dati sole e Plasma - Fase E2".

Topical Editor I. A. Daglis thanks one anonymous referee for her/his help in evaluating this paper.

\section{References}

Balogh, A., Carr, C. M., Acua, M. H., Dunlop, M. W., Beek, T. J., Brown, P., Fornacon, K.-H., Georgescu, E., Glassmeier, K.H., Harris, J., Musmann, G., Oddy, T., and Schwingenschuh, K.: The Cluster Magnetic Field Investigation: overview of in-flight performance and initial results, Ann. Geophys., 19, 1207-1217, 2001, http://www.ann-geophys.net/19/1207/2001/.

Belmont, G. and Chanteur, G.: Advances in magnetopause KelvinHelmholtz instability studies, Phys. Scripta, 40, 124-128, 1989.

Bogdanova, Y. V., Owen, C. J.,Dunlop, M. W., Wild, J. A., Davies, J. A., Lahiff, A. D., Taylor, M. G. G. T., Fazakerley, A. N., Dandouras, I., Carr, C. M., Lucek, E. A., and Rème, H.: Formation of the low/latitude boundary layer and cusp under the northward IMF: simultaneous observations by Cluster and Double Star, J. Geophys. Res., 113, A07S07, doi:10.1029/2007JA012762, 2008.

Chen, S.-H. and Kivelson, M. G.: Nonsinusoidal waves at the magnetopause, Geophys. Res. Lett., 20, 2699-2702, 1993.

Cooling, B. M. A., Owen, C. J., and Schwartz, S. J.: Role of the magnetosheath flow in determining the motion of open flux tubes, J. Geophys. Res., 106, 18763-18775, 2001.

Cowley, S. W. H.: The causes of convection in the Earth's magnetosphere: A review of developments during the IMS, Rev. Geophys. Space Phys., 20, 531-565, 1982.

Crooker, N. U., Siscoe, G. L., and Geller, R. B.: Persistent pressure anisotropy in the subsonic magnetosheath region, Geophys. Res. Lett., 3, 65-68, 1976.

Crooker, N. U.: Reverse convection, J. Geophys. Res., 97, 1936319372, 1992.

Fairfield, D. H., Otto, A., Mukai, T., Kokubun, S., Lepping, R. P., Steinberg, J. T., Lazarus, A. J., and Yamamoto, T.: Geotail observations of the Kelvin-Helmholtz instability at the equatorial magnetotail boundary for parallel northward fields, J. Geophys. Res., 105, 21159-21173, 2000.

Foullon, C., Farrugia, C. J., Fazakerley, A. N., Owen, C. J., Gratton, F. T., and Torbert R. B.: Evolution of Kelvin-Helmholtz activity on the dusk flank magnetopause, J. Geophys. Res., 113, A11203, doi:10.1029/2008JA013175, 2008.

Fuselier, S. A., Klumpar, D. M., and Shelley, E. G.: Ion reflection and transmission during reconection at the Earth's subsolar magnetopause, Geophys. Res. Lett., 18, 139-142, 1991.

Fuselier, S. A., Anderson, B. J., and Onsager, T. G.: Particle signatures of magnetic topology at the magnetopause: AMPTE/CCE observations, J. Geophys. Res., 100, 11805-11821, 1995.

Fuselier, S. A., Anderson, B. J., and Onsager T. G.: Electron and ion signatures of field line topology at the low-shear magnetopause, J. Geophys. Res., 102, 4847-4863, 1997.

Gosling, J. T., Thomsen, M. F., Bame, S. J., Elphic, R. C., and Russell, C. T.,: Observations of reconnection of interplanetary and lobe magnetic field lines at the high-latitude magnetopause, J. Geophys. Res., 96, 14097-14106, 1991.

Hasegawa, H., Fujimoto, M., Phan, T.-D., Rème, H., Balogh, A., Dunlop, M. W., Hashimoto, C., and TanDokoro, R.: Transport of solar wind into Earth's magnetosphere through rolled-up KelvinHelmholtz vortices, Nature, 430, 755-758, 2004.

Hasegawa, H., Fujimoto, M., Takagi, K., Saito, Y., Mukai, T. and Rème, H.: Single-spacecraft detection of rolled-up KelvinHelmholtz vortices at the flank magnetopause, J. Geophys. Res., 111, A09203, doi:10.1029/2006JA011728, 2006. 
Hasegawa, H., Retinò, A., Vaivads, A., Khotyaintsev, Y., André, M., Nakamura, T. K. M., Teh,W.-L., Sonnerup, B. U. Ö, Schwartz, S. J., Seki, Y., Fujimoto, M., Saito, Y., Rème, H., and Canu, P.: Kelvin-Helmholtz waves at the Earth's magnetopause: Multiscale development and associated reconnection, J. Geophys. Res., 114, A12207, doi:10.1029/2009JA014042, 2009.

Hashimoto, C. and Fujimoto, M.: Kelvin-Helmholtz instability in an unstable layer of finite thickness, Adv. Sp. Res., 37, 527-531, 2006.

Hones Jr., E. W., Birn, J., Bame, S. J., Asbridge, J. R., Paschmann, G., Sckopke, N., and Haerendel, G.: Further Determination of the Characteristics of Magnetospheric Plasma Vortices with Isee 1 and 2, J. Geophys. Res., 86(A2), 814-820, 1981.

Johnstone, A. D., Alsop, C., Burge, S., et al.: Peace: A plasma electron and current experiment, Space Sci. Rev., 79, 351-398, doi:10.1023/A:1004938001388, 1997.

Miura, A.: Dependence of the magnetopause Kelvin-Helmholtz instability on the orientation of the magnetosheath magnetic field, Geophys. Res. Lett., 22, 2993-2996, 1995.

Miura, A.: Self-organization in the two-dimensional transverse Kelvin-Helmholtz instability, J. Geophys. Res., 104, 395-411, 1999.

Miura, A. and Pritchett, P. L.: Nonlocal stability analysis of the MHD Kelvin-Helmholtz instability in a compressible plasma, J. Geophys. Res., 87, 7431-7444, 1982.

Nakamura, T. K. M. and Fujimoto, M.: Magnetic reconnection within rolled-up MHD-scale Kelvin-Helmholtz vortices: Twofluid simulations including finite electron inertial effects, Geophys. Res. Lett., 32, L21102, doi:10.1029/2005GL023362, 2005.

Nakamura, T. K. M., Fujimoto, M., and Otto, A.: Magnetic reconnection induced by weak Kelvin-Helmholtz instability and the formation of the low-latitude boundary layer, Geophys. Res. Lett., 33, L14106, doi:10.1029/2006GL026318, 2006.
Nishino, M. N., Fujimoto, M., Ueno, G., Mukai, T., and Saito, Y.: Origin of temperature anisotropies in the cold plasma sheet: Geotail observations around the Kelvin-Helmholtz vortices, Ann. Geophys., 25, 2069-2086, 2007, http://www.ann-geophys.net/25/2069/2007/.

Nykyri, K., Otto, A., Lavraud, B., Mouikis, C., Kistler, L. M., Balogh, A., and Rème, H.: Cluster observations of reconnection due to the Kelvin-Helmholtz instability at the dawnside magnetospheric flank, Ann. Geophys., 24, 2619-2643, 2006, http://www.ann-geophys.net/24/2619/2006/.

Otto, A. and Fairfield, D. H.: Kelvin-Helmholtz instability at the magnetotail boundary, MHD simulation and comparison with Geotail observations, J. Geophys. Res., 105, 21175-21190, 2000.

Rème, H., Aoustin, C., Bosqued, J. M., et al.: First multispacecraft ion measurements in and near the Earth's magnetosphere with the identical Cluster ion spectrometry (CIS) experiment, Ann. Geophys., 19, 1303-1354, 2001, http://www.ann-geophys.net/19/1303/2001/.

Retinò, A., Bavassano Cattaneo, M. B., Marcucci, M. F., Vaivads, A., André, M., Khotyaintsev, Y., Phan, T., Pallocchia, G., Rème, H., Möbius, E., Klecker, B., Carlson, C. W., McCarthy, M., Korth, A., Lundin, R., and Balogh, A.: Cluster multispacecraft observations at the high-latitude duskside magnetopause: implications for continuous and component magnetic reconnection, Ann. Geophys., 23, 461-473, 2005, http://www.ann-geophys.net/23/461/2005/.

Song, P. and Russell, C. T.: Model of the formation of the lowlatitude boundary layer for strongly northward interplanetary magnetic field, J. Geophys. Res., 97, 1411-1420, 1992.

Takagi, K., Hashimoto, C., Hasegawa, H., Fujimoto, M., and TanDokoro, R.: Kelvin-Helmholtz instability in a magnetotail flanklike geometry: Three-dimensional MHD simulations, J. Geophys. Res., 111, A08202, doi:10.1029/2006JA011631, 2006. 